\title{
Estrategias y estilos de aprendizaje y su relación con el rendimiento académico en estudiantes universitarios de Psicología Educativa
}

\author{
Strategies and learning styles and their relationship with \\ academic performance in university students of Educational \\ Psychology
}

\author{
Hilda Josefina Trelles Astudillo ${ }^{1 *}$, Hugo Patricio Alvarado Maldonado ${ }^{1}$ y María Luisa Montánchez Torres ${ }^{1}$ \\ ${ }^{1}$ Universidad Católica de Cuenca \\ *htrellesa@ucacue.edu.ec
}

DOI: https://doi.org/10.26871/killkana_social.v2i2.292

\begin{abstract}
Resumen
La presente investigación tiene por objetivo asociar el rendimiento académico y las estrategias y estilos de aprendizaje empleados por estudiantes del segundo ciclo de Psicología Educativa de la Universidad Católica de Cuenca, de la ciudad de Cuenca, Ecuador. A partir de un enfoque de investigación cuantitativo, se realizó un estudio descriptivo explicativo, siguiendo un diseño no experimental observacional de corte transversal en la Unidad Académica de Pedagogía, Psicología y Educación, Universidad Católica de Cuenca, en Cuenca Ecuador, durante el período octubre 2015-marzo 2016. La muestra seleccionada mediante un muestreo no probabilístico por conveniencia fue de 29 estudiantes de ambos sexos, los cuales cursan el segundo Ciclo de la carrera de Psicología Educativa. Se aplicaron los instrumentos ACRA y CHAEA para la determinación de estrategias y estilos de aprendizaje respectivamente. Se realizó estadística descriptiva, inferencial y de asociación entre las variables mediante pruebas no paramétricas y de correlación. Las estrategias de aprendizaje empleadas son insuficientes, principalmente centradas en la adquisición y codificación de la información sin asociación con el rendimiento académico de las materias evaluadas. Los estilos de aprendizaje predominantes fueron el Activo y el Reflexivo, mostrando una asociación débil con los resultados académicos de las asignaturas cursadas. La baja influencia de las estrategias y estilos de aprendizaje sobre los resultados académicos impone la necesaria contextualización de la enseñanza en función de las actitudes y aptitudes de los estudiantes con vistas a lograr un mayor rendimiento académico.
\end{abstract}

Palabras clave: Estrategias de aprendizaje, estilos de aprendizaje, enseñanza aprendizaje, evaluación de logros.

\section{Abstract}

The aim of this research is to associate academic achievement and learning styles and strategies used by students in the second cycle of educational psychology UCACUE city of Cuenca, Ecuador. From an approach of quantitative research, an explanatory descriptive study, following an observational not experimental design was realized at the Academic Unit of Pedagogy, Psychology and Education, Catholic University of Cuenca in Cuenca Ecuador, during the school year 2015 -2016. Sample was selected through a non-probabilistic convenience sampling its extension was 29 students of both sexes, who enrolled in the second cycle of the career of Educational Psychology. ACRA and CHAEA questionaries' for determining strategies and learning styles were applied respectively. Descriptive, inferential and statistical association between variables was performed using non-parametric tests and correlation. The strategies used are insufficient, mainly focused on the acquisition and encoding of information without association with the academic performance of the subjects evaluated. The predominant learning styles were the Active and Reflexive, showing a weak association with the academic results of the subjects studied. Low influence of Strategies and Learning Styles on academic results makes the necessary contextualization of teaching based on the attitudes and skills of students in order to achieve higher academic achievement.

Key words: Learning style, Learning strategies, academic achievement, teaching learning, achievement evaluation. 


\section{Introducción}

El rendimiento académico constituye uno de los principales indicadores de resultados en la gestión de la docencia en las Instituciones de nivel terciario de enseñanza(Gazzola y Pires, 2008). En la práctica educativa se reportan diversos resultados que generan interrogantes sobre el carácter complejo-multifactorial del aprendizaje y el éxito académico. Algunas de estas situaciones pueden ser las siguientes: estudiantes con resultados académicos relevantes en materias de perfil humanísticos y de ciencias, estudiantes con resultados académicos relevantes en materias de perfil humanístico y no satisfactorio en ciencias o viceversa y estudiantes con resultados académicos no satisfactorios en ambas áreas del conocimiento.

¿Qué causas provocan que estudiantes en similares condiciones socioculturales de enseñanza tengan una diversidad tan grande de resultados académicos?

La respuesta a esta interrogante evidentemente se debe buscar en las características individuales del estudiante en las dimensiones biopsicosocial.

La diversidad de concepciones existentes en las categorías Estilos y Estrategias de Aprendizaje han sido objeto de crítica y visualizados como una barrera para su mejor comprensión y generalización en el ámbito educativo y pedagógico(Valadez Huizar, 2009). Los intentos por sistematizar estas categorías se recogen en diversas publicaciones que revisan las tendencias e intentan llegar a una conciliación entre la enorme dispersión existente(Bahamón Muñetón, Vianchá Pinzón, Alarcón, Liliana, y Bohórquez Olay, 2012; Pantoja Ospina, Duque Salazar, y Correa Meneses, 2013)

Diversas investigaciones han descrito factores influyentes sobre el rendimiento académico(García Ortiz, López de Castro Machado, y Rivero Frutos, 2014; Barahona, 2014; Garzón, Rojas, Riesgo, del Pinzón, y Salamanca, 2010). Entre los más estudiados se encuentran las Estrategias de Aprendizaje (EA) y Estilos de Aprendizaje (ESTA) y los enfoques de aprendizaje(Valle Arias y cols., 2000). Existe una abundante bibliografía que registra investigaciones que han seguido tres líneas principales: a) Análisis de estilos de aprendizaje en diferentes contextos universitarios b) Variables asociadas a estilos y estrategias de aprendizaje c) Relación entre estilos y estrategias de aprendizaje con rendimiento académico.

La conceptualización de EA y ESTA ha sido realizada por una extensa gama de autores con semejanzas y ligeras diferencias, en muchos casos solapando conceptos como estrategias, tácticas y técnicas de aprendizaje(Bahamón Muñetón y cols., 2012). A modo de consenso las EA se definen como el conjunto de actividades conscientes e intencionales que guían las acciones a seguir para alcanzar determinadas metas educativas(Valle Arias, Barca Lozano, González Cabanach, y Núñez Pérez, 1999). Las investigaciones refieren componentes estructurales de las EA en las esferas cognitivas y sociales. Los componentes cognitivos son adquisición, codificación, recuperación; mientras que el apoyo se enmarca en el ámbito social. El uso de las EA está condicionado por variables del estudiante como el propósito o motivación por las tareas, el grado de supervisión de la tarea y los conocimientos previos de la temática. Un aspecto a resaltar es la heterogeneidad de instrumentos empleados en la caracterización de las EA sin predominio de alguno. Entre los más utilizados se encuentran Inventario de estrategias de aprendizaje de Ronald Schmeck(Fernández, Martínez, Macarena, y Araneda, 2009), el MLSQ(Martínez y Galán, 2000).

Otra variable relacionada a los resultados académicos es estilos de aprendizaje, con diversidad de conceptualizaciones e impacto en los resultados académicos(Cabrera Albert y Fariñas León, 2005). La definición de ESTA incluye un sistema complejo de rasgos cognitivos, afectivos y fisiológicos estables, caracterizando cómo los alumnos responden a sus ambientes de aprendizaje. De modo general los individuos responden a un estilo particular y predominante de aprendizaje que le permite interrelacionarse con su ambiente y que implica elementos propios de la cognición y de la personalidad, considerando dos aspectos básicos: 1manera de aprender la percepción y 2 - el procesamiento de la información(Bolívar López J. y Rojas Velásquez, 2008).

Una caracterización de los ESTA, puede resumirse en (González Clavero, 2011):

- Existencia de bases biológicas en los hemisferios cerebrales que condiciona la percepción y el lenguaje

- El aprendizaje es influenciado por la cultura, la experiencia y es susceptible de desarrollo.

- Pueden ser desarrollados como actividad individual (aprendizaje) e institucional (enseñanza) de manera interactiva.

- Deben ser potenciados por las instituciones educativas de modo que se recree un ambiente educativo favorable.

- Combinación de estilos de aprendizaje hasta la consolidación de uno predominante por parte del estudiante.

La clasificación de ESTA es diversa según la fuente del autor consultado, aunque responde a dos criterios principales: formas de percibir la información y formas de procesar la información.

A partir de los planteamientos de Kolb se llega a la propuesta de 4 estilos de aprendizaje principales. En esta clasificación se destaca la inclusión de factores de naturaleza genética, vivenciales y demandas del entorno(Kolb, 1984). Siguiendo este enfoque Peter Honey y Alan Mumford (1986) elaboraron un cuestionario de Estilos de Aprendizaje enfocado al mundo empresarial. Al cuestionario le llamaron LSQ (Learning Styles Questionaire) y con él, pretendían averiguar por qué en una situación en que dos personas comparten texto y contexto una aprende y la otra no. En base de este resultado se presentan cuatro tipos de aprendizaje que a su vez responden al proceso cíclico de aprendizaje: Activo (animador, improvisador, arriesgado, espontáneo), Reflexivo (ponderado, concienzudo, receptivo, analítico y exhaustivo), Teórico (metódico, objetivo, crí- 
tico, estructurado) y Pragmático (experimentador, práctico, directo, eficaz, realista).(Acevedo y Rocha, 2011).

En Ecuador se han realizado investigaciones que describen los estilos de aprendizaje en una muestra de estudiantes de Ingeniería Industrial y Electrónica de la Universidad Técnica del Norte donde se reportaron estilos de aprendizaje predominante como Pragmáticos y Activos.(Cala Aiello, García Riera, y Jaramillo V, 2014; Chávez Arias, 2015; Quillupangui Nieto, 2013)

El objetivo de la presente investigación es relacionar los estilos y estrategias de aprendizaje con los resultados académicos de estudiantes de segundo ciclo de la carrera de Sicología Educativa en la Universidad Católica de Cuenca en el período 2015-2016.

\section{Diseño de Investigación}

A partir de un enfoque de investigación cuantitativo, se realizó un estudio descriptivo explicativo, siguiendo un diseño no experimental observacional de corte transversal en la Unidad Académica de Pedagogía, Sicología y Educación, Universidad Católica de Cuenca (Ecuador), durante el período septiembre 2015-Febrero 2016.

\section{Población y Muestra}

La población universo corresponde a 60 estudiantes de ambos sexos que cursan el Segundo Ciclo de la Carrera de Psicología Educativa de la Universidad Católica de Cuenca. Son tres las extensiones y sedes donde se hayan repartidos; Sede Azogues, extensión Cañar y Sede Matriz. El rango de edades de los estudiantes oscilaba entre 20-21 años.

La muestra fue seleccionada mediante un muestreo no probabilístico por conveniencia siendo escogidos para la investigación 29 estudiantes de la Sede Matriz. Los criterios de inclusión fueron los siguientes:

- Accesibilidad al estudiante, dado que, la investigadora es docente de la Sede Matriz y del ciclo oportuno.

- Seguimiento de los estudiantes por parte de la investigadora, tanto en el primer ciclo como el ciclo analizado.

\section{Variables, Instrumentos y Recolección de datos}

Las variables estudiadas correspondieron al rendimiento académico, estrategias de aprendizaje y estilos de aprendizaje. El Rendimiento académico fue estimado a partir de los resultados obtenidos en la evaluación sumativa plasmada en los documentos rectores del proceso de enseñanza aprendizaje de cuatro asignaturas cursadas (los estilos de aprendizaje fueron cuantificadas a partir del Cuestionario Honey Alonso de Estilos de Aprendizaje (CHAEA)(Alonso, Gallego, y Honey, 1994) con cuatro categorías como Activo, Reflexivo, Teórico y Pragmático (experimentador, práctico, directo, eficaz, realista). La identificación de las estrategias de aprendizaje empleó el cuestionario ACRA (Román Sánchez y Sagrario Gallego, 1994) categorizadas en adquisición, codificación, recuperación y apoyo según la propuesta de Román Sánchez \&
Rico (1994). Ambos cuestionarios emplearon escala Likert para cuantificar la frecuencia de aparición de las propuestas realizadas en cada uno de los ítems.

La aplicación de los test fue realizada por única vez en la cuarta semana del segundo ciclo de la carrera de Psicología Educativa. Su resolución se llevó a cabo en el entorno docente en condiciones adecuadas de iluminación, temperatura y confort inmobiliario. Este test fue aplicado en forma grupal sin límite de tiempo con una duración de 45 minutos.

\section{Métodos Estadístico -Matemáticos}

El procesamiento estadístico - matemático de los datos fue realizado mediante el programa informático SPSS 23.0 para Windows. Se realizó el análisis de frecuencia para los valores obtenidos en las variables estudiadas y sus dimensiones. La estadística descriptiva reflejó medidas de tendencia central, posición y dispersión como la media, desviación estándar y percentiles. Se empleó métodos de correlación para la asociación entre las variables estudiadas como el coeficiente de regresión de Pearson para variables cuantitativas y la prueba Eta de asociación entre variables cuantitativas y cualitativas nominal. La prueba Chi cuadrado fue usada para determinar diferencias entre las proporciones para las variables cualitativas. El test de KruskalWallis, Friedman y la prueba-t no paramétrica para casos independientes determinó las diferencias entre las variables cuantitativas según grupos existentes.

\section{Procedimientos éticos}

El estudio realizado cumplió con los requisitos mínimos para la investigación con seres humanos. Se concertó la debida autorización de la Unidad Académica y se realizó un consentimiento informado de la investigación a las autoridades y estudiantes participantes mediante comunicación oral y escrita.

\section{Resultados}

La muestra de estudiantes correspondió con 29 estudiantes, 19 de sexo femenino $(65,5 \%)$ sin diferencias en las proporciones de ambos grupos $(\mathrm{X} 2=2,793$; $\mathrm{p}=0,095)$

La figura 1 refleja la estadística descriptiva de la variable Estrategias de aprendizaje y sus dimensiones, obtenidas a partir del cuestionario ACRA. 


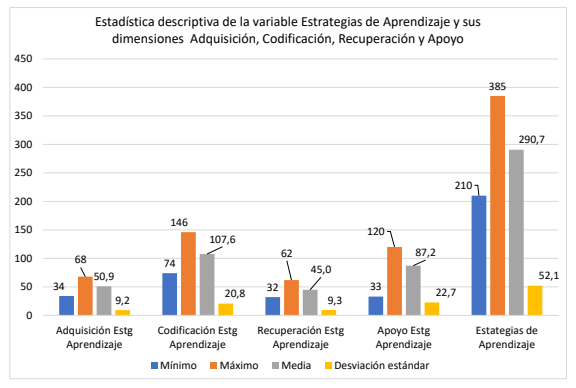

Figura 1. Estadística descriptiva de la variable Estrategias de Aprendizaje y sus dimensiones: Adquisición, Codificación, Recuperación y Apoyo en estudiantes de la carrera de Psicología Educativa, segundo ciclo (UCACUE 2016)

Los valores obtenidos son ligeramente inferiores a los reportados por Román y Sánchez en la caracterización del instrumento ACRA en estudiantes de Secundaria en Valladolid España (Román Sánchez \& Sagrario Gallego, 1994), aunque con mayores diferencias que los encontrados en estudiantes universitarios (Rossi Casé, Neer, Lopetegui, \& Doná, 2010). La magnitud de la variable y las dimensiones oscilan entre el 15 y 45 percentil para el baremo del ACRA. (Adquisición 30 \%; Codificación 45\%; Recuperación $25 \%$ y Apoyo $15 \%$ ).

La comparación de los resultados con lo reportado por Román y colaboradores mostró similitudes para las dimensiones Adquisición ( $\mathrm{t}=-1,998$; $\mathrm{p}=0.056$; Varianzas diferentes) y Codificación ( $\mathrm{t}=-0,659 ; \mathrm{p}=0.515$; Varianzas diferentes). Sin embargo las dimensiones restantes mostraron diferencias significativamente inferiores a las reportadas por el baremo del instrumento ACRA (Recuperación

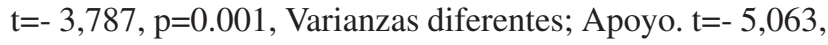
$\mathrm{p}=0.000$, Varianzas similares).

La aplicación del CHAEA para la identificación de los Estilos de Aprendizaje del grupo estudiado se muestra en la figura 2. Los estilos de aprendizaje predominantes se corresponden con el Activo y Reflexivo siendo el 79,6\% de la muestra $\left(\mathrm{X}^{2}=10,586 ; \mathrm{p}=0,014\right)$. El resto de los estilos fueron minoritarios.

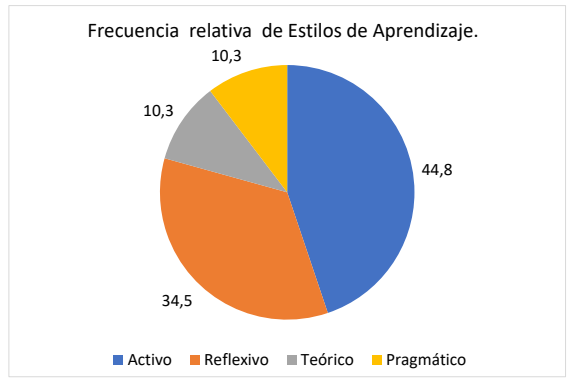

Figura 2. Análisis de frecuencia de la variable Estilos de Aprendizaje y sus categorías en estudiantes de la carrera de Psicología Educativa, Segundo ciclo, UCACUE 2016.
Los resultados académicos se muestran en la tabla 1.

Tabla 1 Estadísticos descriptivos de los resultados académicos por materia cursada en estudiantes de Sicología Educativa de la UCACUE 2015-2016

\begin{tabular}{lccccc}
\hline Items & N & Mínimo & Máximo & Media & $\begin{array}{c}\text { Desviación } \\
\text { estándar }\end{array}$ \\
\hline $\begin{array}{l}\text { Nota Lengua y Lite- } \\
\text { ratura }\end{array}$ & 28 & 71 & 99 & 86,00 & 5,925 \\
$\begin{array}{l}\text { Nota Historia y Fi- } \\
\text { losofía }\end{array}$ & 29 & 70 & 99 & 81,45 & 7,429 \\
$\begin{array}{l}\text { Nota Antropología } \\
\text { Social }\end{array}$ & 29 & 34 & 92 & 79,83 & 10,593 \\
$\begin{array}{l}\text { Nota Psicología y } \\
\text { Escuelas Psicológi- } \\
\text { cas }\end{array}$ & 29 & 70 & 96 & 78,69 & 7,026 \\
\hline
\end{tabular}

La descripción de las medias obtenidas en la puntuación de cada materia difiere de manera significativa para las diferentes asignaturas (Prueba de Friedman $X^{2}=26,443$; $\mathrm{p}=0,000)$. Las puntuaciones menores fueron obtenidas en Antropología Social, Psicología y Escuelas Psicológicas sin diferencias entre estos valores $(\mathrm{T}=0,656 ; \mathrm{p}=0,517)$.

La descripción de los resultados académicos en las diferentes asignaturas según los estilos de aprendizaje empleados se muestra en la figura 3.

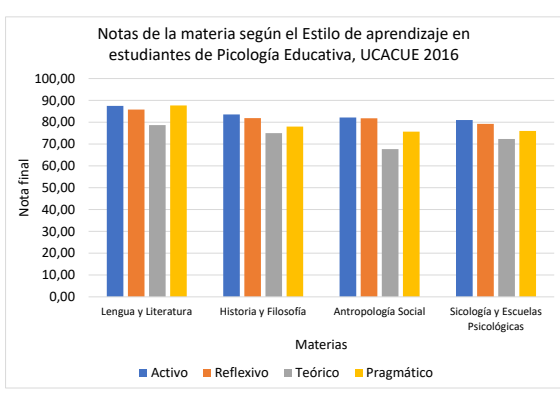

Figura 3.

No se encontraron diferencias en la puntuación alcanzada en las materias según el Estilo de Aprendizaje (Tabla 2).

Tabla 2 Prueba no paramétrica de comparación de notas académicas según Estilos de Aprendizaje.

\begin{tabular}{ccccc}
\hline & $\begin{array}{c}\text { Nota } \\
\text { Lengua y } \\
\text { Literatura }\end{array}$ & $\begin{array}{c}\text { Nota } \\
\text { Historia y } \\
\text { Filosofía }\end{array}$ & $\begin{array}{c}\text { Nota An- } \\
\text { tropología } \\
\text { Social }\end{array}$ & $\begin{array}{c}\text { Nota } \\
\text { Sicología } \\
\text { y Escuelas } \\
\text { Psicológi- } \\
\text { cas }\end{array}$ \\
\hline Chi-cuadrado & 2,850 & 3,613 & 2,794 & 4,944 \\
gl & 3 & 3 & 3 & 3 \\
Sig. asintótica & 0,415 & 0,306 & 0,425 & 0,176 \\
\hline a. Prueba de Kruskal Wallis; b. Variable de agrupación: Estilos de Aprendizaje \\
\hline
\end{tabular}

Sin embargo, al analizar la asociación entre la variable notas para cada materia y el estilo de aprendizaje empleado 
por los estudiantes mediante la prueba de asociación Eta, se encontró una asociación débil, moderada o fuerte según el tratamiento de la variable como dependiente o independiente (tabla 3).

Tabla 3 Prueba no paramétrica de comparación de notas académicas según Estilos de Aprendizaje.

\begin{tabular}{lcc}
\hline \multirow{2}{*}{ Materia } & \multicolumn{2}{c}{ Prueba ETA: Valor Estadístico de la prueba } \\
& Variable dependiente a & Variable independiente b \\
\hline Lengua y Literatura & 0,457 & 0,830 \\
Historia y Filosofía & 0,375 & 0,809 \\
Antropología Social & 0,437 & 0,717 \\
$\begin{array}{l}\text { Sicología y Escue- } \\
\text { las Psicológicas }\end{array}$ & 0,393 & 0,847 \\
\hline
\end{tabular}

a- La materia es considerada variable dependiente con respecto a los Estilos de aprendizaje

b- La materia es considerada variable independiente con respecto a los Estilos de aprendizaje

La asociación entre las estrategias de aprendizaje y los resultados académicos se realizó a través de la correlación de Pearson. No se evidenció asociación entre las estrategias de aprendizaje y sus dimensiones con los resultados académicos de las diferentes materias cursadas (datos no mostrados), aunque al controlar la variable estilos de aprendizaje se encontró una correlación débil entre los resultados académicos de la materia Antropología Social y las estrategias de aprendizaje $(\mathrm{R}=0,390 ; \mathrm{p}=0,040)$

\section{Discusión}

Los resultados obtenidos coinciden con estudios previos de la región (Bahamón Muñetón, Vianchá Pinzón, Alarcón Alarcón, y Bohórquez Olaya, 2013). El análisis de la Estrategias de aprendizaje reveló un déficit en la muestra estudiada. Reportes previos de otras investigaciones muestran valores superiores para todas las dimensiones en estudiantes universitarios de diferentes países como Argentina y España (Rossi Casé, Neer, Lopetegui, y Doná, 2010). Esto sugiere que las estrategias de aprendizaje están conectadas con las necesidades y preferencias de los estudiantes, de allí la necesidad de apoyar a los estudiantes para que desarrollen habilidades metacognitivas y de forma autónoma construyan su propio aprendizaje.

El desarrollo de las dimensiones indagadas mediante el instrumento ACRA confirma un déficit en la adquisición, codificación, recuperación y apoyo al compararse con grupos etarios similares en la actividad académica. Este resultado contradice afirmaciones previas donde se enfatiza un mayor uso de las estrategias de aprendizaje por parte de estudiantes de carreras de Humanidades (Camarero, Martín, y Herrero, 2000). Una posible explicación puede involucrar al nivel o ciclo académico cursado, tratándose la muestra de estudiantes noveles de segundo ciclo de la carrera de Psicología Educativa.

La ausencia de relación entre las estrategias de aprendizaje y los resultados académicos también difiere de investigaciones previas donde se ha reportado relación directa con los resultados académicos en diferentes áreas del conocimiento como Humanidades(Esguerra y Guerrero, 2010), Matemáticas (Barros, 2007)y Ciencias de la Salud (Díaz y cols., 2009). La Estrategias de aprendizaje contribuyen con la adquisición de información de manera precisa y certera mediante la optimización de la atención y la concentración de los estudiantes, la exploración adecuada de las fuentes de información suministrada en los materiales docentes y la fragmentación de textos usando técnicas como el subrayado, resumen y la significación. Además se favorece el paso de la información a la memoria a largo plazo mediante la repetición en diferentes variantes. También incrementa la codificación de la información a través de recursos nemotécnicos, de elaboración y de organización, siendo estos los de mayor influencia en el almacenamiento de la información y determinantes en el logro de resultados académicos relevantes.

Las dimensiones de recuperación y apoyo mostraron el mayor déficit en la muestra, lo que sugiere una escasa sistematización en el proceso de enseñanza-aprendizaje. Esta situación limita la búsqueda de alternativas para la evocación de conceptos, ideas, palabras y la generación de una respuesta adecuada a la situación planteada. De manera concomitante el déficit de apoyo puede traducirse en debilidades metacognitivas de autoconocimiento y socio afectivas - motivacionales que impactan en la evaluación de los logros alcanzados, el reordenamiento o replanteamiento de nuevas formas de aprendizaje y el control de estados afectivos negativos, como la ansiedad y el estrés de modo que optimicen los resultados académicos.

Un hecho curioso es que al controlar la variable estilos de aprendizaje se apreció una relación débil entre estrategias de aprendizaje y resultados académicos $(\mathrm{R}=0,390$; $\mathrm{p}=0.041$ ) corroborando la sinergia entre las variables estilos y estrategias de aprendizaje en el logro de resultados académicos satisfactorios.

Lo anterior sugiere una profunda revisión de la planificación del proceso de enseñanza-aprendizaje que fomente la enseñanza de las estrategias de aprendizaje en los estudiantes, logrando una verdadera apropiación como parte de su desarrollo psicosocial.

Coincidiendo con Ruiz, Trillos y Morales (2006) uno de los Estilos de aprendizaje predominantes en la muestra fue el reflexivo (Ruiz y cols., 2006), aunque difieren los hallazgos en la prevalencia del estilo activo, en comparación con el estilo teórico o pragmático de otras investigaciones (Madrid Valdebenito, Acevedo, Chiang Salgado, Montecinos, y Reinicke Seiffert, 2009), sin relación con el tipo de carrera cursada (Humanidades vs Ciencias) .

Los estudios de estilos de aprendizaje reportados muestran una gran dispersión de datos y resultados con la imposibilidad de hacer una generalización. La literatura recoge un gran número de variables que pueden estar o no asociadas con los estilos de aprendizaje empleados por los estudiantes. Algunas de ellas son el sexo biológico, el tipo de carrera, el curso del estudiante y el ciclo al 
que pertenece. En la literatura consultada no se menciona asociación o diferencias según el tipo de materia cursada.

Los resultados presentados no mostraron diferencias de los estilos de aprendizaje según la materia cursada, quizás debido a que todas pertenecen al área de humanidades e impartidas por docentes con orientaciones metodológicas similares por el comité de carrera. La no contrastación de diferencias de resultados académicos entre los diferentes estilos de aprendizaje para todas las materias sugiere que esta variable no es determinante y solo explica una porción de los resultados, lo que fue corroborado mediante la asociación débil de todas las categorías y los resultados docentes.

Un aspecto relevante es el carácter dinámico de los estilos de aprendizaje, estando influenciados por un conjunto de variables biopsicosociales como desarrollo neurocognitivo, edad, sexo, hábitos, medio social y entorno. Lo anterior se traduce en que un individuo puede combinar o transitar por diversos estilos de aprendizaje durante su vida hasta consolidar uno de manera preferencial (Pantoja Ospina y cols., 2013). Entonces queda de parte de la Universidad orientar y mostrar los métodos y técnicas adecuadas de acuerdo a las condiciones biopsicosociales del estudiante.

Para influir en los resultados académicos desde los estilos de aprendizaje es imprescindible la reorientación del proceso de enseñanza en función de un diagnóstico certero realizado por docentes según las características de la masa estudiantil, sus preferencias y la didáctica específica de las ciencias a emplear. De manera irresoluta el proceso de enseñanza-aprendizaje debe incluir espacios y tareas de aprendizaje que integre la experiencia concreta, la observación reflexiva, la conceptualización abstracta y la experimentación activa con el fin de lograr un acercamiento al conocimiento desde todos los estilos mostrando al estudiante la necesidad de todas las dimensiones para llegar a una apropiación definitiva y significativa del conocimiento

¿Cómo influir en estrategias y estilos de aprendizaje para lograr mejores rendimientos académicos?

El desarrollo de las estrategias y los estilos de aprendizaje responde a diversos factores relacionados con las características personológicas del estudiante así como al proceso educacional donde esté inmerso. Los resultados anteriores sugieren una planificación del proceso docente de modo que incluya espacios de aprendizaje y tareas docentes que vinculen las estrategias y los estilos de aprendizaje contextualizados a los núcleos del conocimiento incluidos a nivel curricular. Esta consecución se logrará mediante una adecuada articulación y desarrollo armónico y sincrónico de las demandas del proceso docente y el desarrollo de la personalidad del estudiante. Solo la sistematización sistémica de estas categorías logrará un incremento en la eficacia del proceso docente y mayores logros de aprendizaje en los estudiantes.

\section{Conclusiones}

Las estrategias de aprendizaje en estudiantes del segundo ciclo de la carrera de Psicología Educativa de la UCACUE son deficitarias sin completar el ciclo necesario para el aprendizaje significativo.

Los Estilos de aprendizaje predominante son el Activo y Reflexivo.

La no correlación entre Estrategias de Aprendizaje o la asociación moderada de los Estilos de Aprendizaje con los resultados académicos impone la necesaria contextualización de la enseñanza en función de las actitudes y aptitudes de los estudiantes con vistas a lograr un mayor rendimiento académico.

\section{Referencias Bibliográficas}

Acevedo, C., y Rocha, F. (2011). Estilos de Aprendizaje, género y rendimiento académico. Revista Estilos de Aprendizaje, 8(8), 1-16.

Alonso, C. M., Gallego, D. J., y Honey, P. (1994). Los Estilos de Aprendizaje: Procedimientos de Diagnóstico y Mejora. Bilbao,: Ediciones Mensajeros.

Bahamón Muñetón, M. J., Vianchá Pinzón, M. A., Alarcón, A., Liliana, L., y Bohórquez Olay, C. I. (2012). Estilos y estrategias de aprendizaje: una revisión empírica y conceptual de los últimos diez años. Pensamiento Psicológico, 10(1), 129-144.

Bahamón Muñetón, M. J., Vianchá Pinzón, M. A., Alarcón Alarcón, L. L., y Bohórquez Olaya, C. I. (2013). Estilos y estrategias de aprendizaje relacionados con el logro académico en estudiantes universitarios. Pensamiento Psicológico, 11(1), 115-129.

Barahona, P. (2014). Factores determinantes del rendimiento académico de los estudiantes de la Universidad de Atacama. Estud. pedagóg. [online], 40(1), p.25-39.

Barros, A. (2007). Estrategias de aprendizaje en las matemáticas que emplean los estudiantes universitarios. Revista Perspectiva Psicológica(9), 37-51.

Bolívar López J. y Rojas Velásquez, F. (2008, diciembre). Los estilos de aprendizaje y el locus de control en estudiantes que inician estudios superiores y su vinculación con el rendimiento académico. Investigación y Postgrado, 23(3), 199-215.

Cabrera Albert, J. S., y Fariñas León, G. (2005). El estudio de los estilos de aprendizaje desdwe una perspectiva vigotsquiana: una aproximación conceptual. Revista Iberoamericana de Educación, 37(1), 1-9.

Cala Aiello, R., García Riera, M., y Jaramillo V, M. (2014). Determinación de los estilos de aprendizaje de estudiantes de 1er curso de ing. Industrial y electrónica de la universidad técnica del norte. Ibarra. Ecuador. Revista de Estilos de Aprendizaje pp, 7(14), 43-67.

Camarero, F., Martín, F., y Herrero, J. (2000). Estilos y estrategias de aprendizaje en estudiantes universitarios. Psicothema, 12(4), 615-622.

Chávez Arias, L. (2015). Relación entre las estrategias de aprendizaje de los estudiantes de ingeniería me- 
cànica de la espoch y su rendimiento académico en la asignatura análisis matemático (Inf. Téc.). Riobamba, Ecuador.

Díaz, A., Mora, A., La fuente, E., Gargiulo, B., Bianchi, B., Terán, C., y Escanero, F. (2009). Estilos de aprendizaje de estudiantes de medicina en universidades latinoamericanas y españolas: relación con los contextos geográficos y curriculares. Revista Educación Médica, 12(3), 183-194.

Esguerra, P., y Guerrero, P. (2010). Estilos de aprendizaje y rendimiento académico en estudiantes de psicología. Revista Divérsitas Perspectiva Psicológica, 6(1), 97109.

Fernández, G., Martínez, C., Macarena, M., y Araneda, R. (2009). Estrategias de aprendizaje y autoestima: su relación con la permanencia y deserción universitaria. Revista de Estudios Pedagógicos, 35(1), 27-45.

García Ortiz, Y., López de Castro Machado, D., y Rivero Frutos, O. (2014). Estudiantes universitarios con bajo rendimiento académico, ¿qué hacer? EDUMECENTRO, 62(2), 272-278.

Garzón, R., Rojas, M. O., Riesgo, L., del Pinzón, M., y Salamanca, A. L. (2010). Factores que pueden influir en el rendimiento académico de estudiantes de Bioquímica que ingresan en el programa de Medicina de la Universidad del Rosario-Colombia. Educación Médica, 13(2), 85-96.

Gazzola, A. L. Y., y Pires, S. (2008). Hacia una política regional de aseguramiento de la calidad en educación superior para América Latina y el Caribe. (Inf. Téc.). Caracas:: IESALC/UNESCO.

González Clavero, M. V. (2011, abril). Estilos de aprendizaje: su influencia para aprender a aprender. Revista Estilos de Aprendizaje, 7(7), 12-26.

Kolb, D. (1984). Experiential learning experiences as the source of learning development. Nueva York: Prentice Hall.

Madrid Valdebenito, V., Acevedo, C. G., Chiang Salgado, M. T., Montecinos, H., y Reinicke Seiffert, K. (2009). Perfil de estilos de aprendizaje en estudiantes de primer año de dos carreras de diferentes areas en la Universidad de Concepción. Revista Estilos de Aprendizaje, $n^{\circ} 3$, Vol 2, , 2(3), 57-69.

Martínez, J., y Galán, F. (2000). Estrategias de aprendizaje, motivación y rendimiento académico en alumnos universitarios. Revista Española de Orientación y Psicopedagogía, 11(19), 35-50.

Pantoja Ospina, M. A., Duque Salazar, L. I., y Correa Meneses, J. S. (2013). Modelos de estilos de apren- dizaje: una actualización para su revisión y análisis. Revista Colombiana de Educación(64), 79-105.

Quillupangui Nieto, R. P. (2013). "Los estilos de aprendizaje y su incidencia en el rendimiento académico de los estudiantes del centro de educación básica dr. Luis Eguiguren de la parroquia de Amaguaña cantón Quito provincia Pichincha" (Trabajo de Investigación Previa a la obtención del Grado Académico de Magíster en Diseño Curricular y Evaluación Educativa). Quito.

Román Sánchez, J. M., y Sagrario Gallego, R. (1994). ACRA. Escalas de Estrategias de Aprendizaje. Madrid. Madrid: TEA Ediciones.

Rossi Casé, L. E., Neer, R. H., Lopetegui, M. S., y Doná, S. (2010). Estrategias de aprendizaje y rendimiento académico según el género en estudiantes universitarios. SRevista de Psicología, 11, 199-211.

Ruiz, B., Trillos, J., y Morales, J. (2006). Estilos de aprendizaje y rendimiento académico en estudiantes universitarios. Revista Galegoportuguesa de Psicoloxía e Educación(13), 441-460.

Valadez Huizar, M. (2009, diciembre). Estilos de aprendizaje y estilos de pensamiento:precisiones conceptuales. Revista de Educación y Desarrollo, , 11, 19-30.

Valle Arias, A., Barca Lozano, A., González Cabanach, R., y Núñez Pérez, J. C. (1999). Las estrategias de aprendizaje revision teorica y conceptual. Revista Latinoamericana de Psicología, 31(3), 425-461.

Valle Arias, A., González Cabanach, R., Núñez Pérez, j. C., Suárez Riveiro, J. M., Piñeiro Aguín, I., y Rodríguez Martínez, S. (2000). Enfoques de aprendizaje en estudiantes universitarios. Psicothema, 12(3), 368-375.
Recibido: 18 de junio de 2018

Aceptado: 29 de junio de 2018 
